\title{
X-RAY EVIDENCE FOR MULTIPLE ABSORBING STRUCTURES IN SEYFERT GALAXIES*
}

\author{
J. M. GELBORD \\ MIT Center for Space Research, NE80-6091 \\ 77 Massachusetts Ave. \\ Cambridge, MA 02139, USA \\ E-mail: jonathan@space.mit.edu
}

K. A. WEAVER AND T. YAQOOB

\begin{abstract}
We have used X-ray spectra to measure attenuating columns in a large sample of Seyfert galaxies. Over 30 of these sources have resolved radio jets, allowing the relative orientation of the nucleus and host galaxy to be constrained. We have discovered that the distribution of absorbing columns is strongly correlated with the relative orientation of the Seyfert structures. This result is inconsistent with unification models having only a torus and is instead most readily explained if a second absorber is included: in addition to a Compton-thick torus there would also be a larger-scale absorber with $N_{H}<10^{23} \mathrm{~cm}^{-2}$. The second absorber is aligned with the host galactic plane while the torus is arbitrarily misaligned.
\end{abstract}

The canonical unified model for Seyfert galaxies invokes chance lineof-sight obscuration by a single parsec-scale torus to explain different observed phenomena. However, some data are better explained by a model incorporating a second absorbing structure ${ }^{2,3,4}$. We test this dual-absorber (DA) model, assuming one absorber is the canonical parsec-scales torus, arbitrarily misaligned with the host galactic plane, while the other is at 100-pc scales, aligned with the host galaxy disc (hereafter the galacticaligned absorber, or GA). Furthermore, we assume the torus is Compton thick $\left(N_{H}>10^{23.5} \mathrm{~cm}^{-2}\right)$ and the GA has a lower attenuating column. Either absorber is capable of obscuring the central engine.

The relative alignment of the two obscuring structures plays an important role in the DA model. Compton-thin Seyfert $2 \mathrm{~s}$ will be observed only if the line of sight intercepts the GA and avoids the torus. If the torus and

*Drawn from Chap. 4 of Gelbord $2002^{1}$, wherein more details and references may be found. Online at http://space.mit.edu/ jonathan/papers/thesis/abstract.html. 


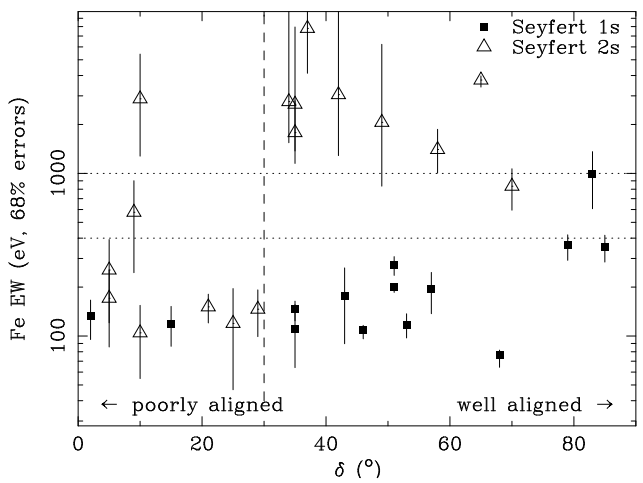

Figure 1. Compton-thick sources have EW $>1 \mathrm{keV}$, and Compton-thin ones have EW $<400 \mathrm{eV}$. As predicted, Compton-thin Seyfert 2s (GA-only absorption) are only found in strongly misaligned systems. The distribution of all Compton-thin sources (incl. type 1s) is insensitive to $\delta$, as expected if only the orientation of the torus is important.

GA are well aligned much or all of the GA will lie within the shadow cast by the torus, so randomly-oriented lines of sight are likely to either intercept both or neither, hence most well-aligned Seyferts would be observed as either unabsorbed type $1 \mathrm{~s}$ or Compton-thick type $2 \mathrm{~s}$. On the other hand, when the absorbers are misaligned the GA covers part of the opening of the torus, leaving fewer sight lines with a direct view of the nucleus and more sight lines with Compton-thin (GA-only) absorption.

To test the DA model, we choose Seyferts for which the relative alignment can be constrained and use X-ray spectra to discriminate between Compton-thick and Compton-thin attenuation. Alignment is indicated by the $\delta$ values ${ }^{\mathrm{a}}$ of Kinney et al. ${ }^{5}$; small values indicate strong misalignments. $\mathrm{Fe} \mathrm{K} \alpha$ line equivalent width is measured to constrain absorbing columns ${ }^{\mathrm{b}}$. Our measurements (Fig. 1) match the predictions of the DA model.

\section{References}

1. J. Gelbord, PhD Thesis, Johns Hopkins Univ. (2002). Available online at http://space.mit.edu/ jonathan/papers/thesis/abstract.html.

2. K. K. McLeod, \& G. H. Rieke, ApJ 441, 96 (1995).

3. H. Schmitt et al., ApJ 555, 663 (2001).

4. G. Matt, $A \mathscr{E} A$ 355, L31 (2000).

5. A. L. Kinney et al., ApJ 537, 152.

\footnotetext{
${ }^{a} \delta$ is the angle between the (projected) radio jet and major axis of the host galaxy. ${ }^{\mathrm{b}} \mathrm{Fe} \mathrm{K} \alpha \mathrm{EW}$ provides a more robust indicator than measuring $N_{H}$ from continuum modeling because values outside the range $10^{22}-10^{24} \mathrm{~cm}^{-2}$ are not well constrained.
} 